\title{
Creating the Best Possible Health Education Cover Letter and Resume
}

\author{
Sara B. Oswalt \\ University of Texas at San Antonio
}

\begin{abstract}
This article provides an overview of job application strategies for the field of health education. Employment in a non-academic setting is emphasized. Tips for cover letter and resume writing are highlighted.
\end{abstract}

(C) 2005 Californian Journal of Health Promotion. All rights reserved.

Keywords: Health Education, Employment, Professional Development, Public Health

Looking for a job can be an exciting and challenging project, especially when you are already employed or have some financial security. Unfortunately, many times we are looking for a position when it is needed: a recent graduation, being part of a budget cut, or being miserable in the current position. The following article is designed to provide suggestions for writing the best possible cover letter and resume in the field of health education.

Within the field of health education, there are many different types of positions; the style of the resume and cover letter may vary based on the job. An individual applying for an academic position usually uses a curriculum vitae which differs in format and language than a resume, commonly used for community, school, medical and worksite positions. Because of these differences, this article will focus specifically on developing job applications for non academic positions.

\section{Application Packet}

A job application usually consists of two parts: cover letter and resume. These components have different functions and styles, and both are important elements of the application packet. The cover letter and resume should be modified for each job. While some skills are applicable for all jobs, taking the time to tailor your application packet demonstrates a strong interest in that particular job, and employers are more interested in someone who is interested in them.
For the four main areas of health education: community, worksite, school and medical, resumes and cover letters should be different. For example, criteria for worksite health positions may emphasize increased productivity and decreased employee sick time; whereas, community health positions may require more skills related to operating on a limited (or no) budget and working with community coalitions. Individuals working in non-profit organizations have demonstrated specific characteristics such as deep concern for others, high ethics, and enjoy feeling good about their vocation (Thomas, 1998). Understanding these differences and accentuating your skills as appropriate when you apply for various jobs will be invaluable. By using terminology and highlighting skills that resonate with that emphasis area, your resume will stand out as well as demonstrate a strong interest in the position.

\section{The Cover Letter}

Traditionally, the cover letter serves as an introduction; however, if crafted carefully, it can be so much more. The cover letter is a document that contains a different writing style than the resume; it should be in paragraph form and as a result, it provides the reviewer with a sample of your writing skills. A clearly worded, strong document can distinguish your application from others that may be unorganized and contain poorly structured sentences. 
Specifically, the cover letter should explain your interest in that position at that specific company, how you fit with that company, and how you qualify for the position (The College of Wooster, n.d.). The letter should be approximately three to five paragraphs: an introductory paragraph, the body and a concluding paragraph. The entire letter is important; however, the body of the letter is probably the most important as it provides an opportunity to tell the employer what skills and potential you can bring to their organization. While repeated content from the resume should be kept to a minimum, highlight components critical to the job description, previous accomplishments and skills.

Similarly, the cover letter can be used to explain something potentially confusing to the reviewer. For example, while certification for health educators (specifically the certified health education specialist [CHES]) is becoming more common, employers may not understand and appreciate the qualifications of a CHES. One can use the cover letter to briefly inform the reviewer of this certification.

In addition, the cover letter can address an item on the job description that you may not have direct experience in. For instance, diversity is a critical component in most jobs today; however, you may not have any experience in a diversity group or be able to provide examples from school or work to address this component in your resume. A simple solution is to include your perspective on the importance of diversity (or whatever component you are lacking) in the cover letter. The reviewer notices the component is addressed and is not left wondering about your qualifications related to it.

The concluding paragraph summarizes your interest in the position and organization and refers the reader to the resume for more information. In addition, provide at least one method to contact you (usually phone and/or email) and express an interest to provide further information at an interview or other opportunity. Close the letter by thanking the reader for his/her time and consideration.
Be aware that if you are applying for a job online or via email, a one-page cover letter will appear extremely long as an email message. Consider submitting the cover letter and resume as attachments to an email to avoid this potential problem.

\section{The Resume}

The purpose of the resume is to advertise your qualifications enough to obtain an interview. Because the goal is to get an interview, the resume does not need to include everything, but it must include enough to entice the reader into wanting to learn more. As a result, the resume should be more than just a life history. A dry listing of work and educational experiences is not likely to distinguish your resume from the masses, especially if you are a recent graduate with little or no work experience.

\section{First Components}

Name and contact information is usually the first items listed on any resume. While this is basic information such as name, address, telephone numbers, and email addresses, be sure that this information is correct. A typographical error in this section can be extremely damaging. In addition, telephone numbers should have an answering machine or voice mail, and email addresses should be checked on a daily basis. Nothing will make an employer more frustrated than trying to "track down" a candidate. Usually, they will not try very hard, and the application will be discarded.

Because resumes are initially reviewed very briefly, the top half of resume is critical to selling you and your skills (Rockport Institute Ltd., 2003). After contact information, the objective and summary should be listed. The objective is not simply a statement that you are looking for position; the reader needs to be engaged and in order for that to happen, the objective must be unique from others.

The objective is a way to present yourself to the organization that initially highlights your fit with the organization and the specific goals of this position. It is not about your needs or what you desire in a position; consider what the organization would be looking for. One example for an objective is to list the type of position and 
identify two qualities that could be well utilized in that position (Rockport Institute Ltd., 2003). For example "A mid-level worksite health education position in an organization seeking an individual with high energy, excellent motivational skills and an ability to demonstrate an impact on the financial success of the organization." This objective is not about what the applicant is looking for, but connects the reader with the organization's needs and how this applicant meets those needs. As with the cover letter, the objective should be adapted to fit each particular job and organization.

After the objective, a summary of qualification is listed. This summary provides an overview of who you are and what you can offer; it should be direct, clear and convincing. Short statements that highlight your professional experience, qualities and achievements are included to convince the reader why you are the best candidate for the position. One example could be: "Health educator with high interest in diverse populations and 10 years of assessment, program planning and implementation, and evaluation experience. Exemplary organization skills support multi-tasking and managerial responsibilities. Extensive grant writing experience exemplifies analytical, writing and budgeting skills.” The summary also needs to be tweaked for each position.

Some individuals may prefer to combine the objective and the summary sections. For individuals who have been in the same field for an extended period of time and on the same career path, this may be a good option. However, when combining, watch the length to ensure that the section does not become too long and hard to read; consider using bullets or paragraph breaks to ensure that the reviewer can follow.

\section{Resume Content \& Format}

There are three basic formats of resumes: the chronological, the functional and the hybrid (Kennedy, 2003). The chronological resume is the traditional structure of resume: each job (or the last several jobs) is listed in reverse chronological order with the responsibilities for each job detailed. This resume structure does not allow one to highlight their accomplishments and can be especially difficult to use for recent graduates with a limited employment history.

A functional resume format highlights skills and accomplishments. Instead of listing previous positions, major skills are identified and detailed examples are provided. For a health educator, skill categories could include: Assessment, Program Planning, Evaluation, Curriculum Development, Coalition Building, Grant Writing, and Program Implementation. For each skill listed, several examples would be provided. Generic job skills such as communication, managerial, and budget coordination could be listed. After the skills section, an employment history is summarized but no job descriptions are provided. This resume structure can be especially helpful to health educators who have done a lot of volunteer or part-time work. Likewise, individuals who have taken time off or who are switching into the field of health education may also find this format useful. While this type of resume features your assets, it can leave the reviewer wondering exactly what you did in each job.

A hybrid resume format - which includes both chronological and functional components encompasses the advantages of both structures. One can list skills and accomplishments before listing jobs with brief descriptions or one could list employment history providing highlights and accomplishments for individual positions. There are two disadvantages of this format; the resume may be too lengthy and it may appear repetitious if information is included in both the chronological and function areas. However, careful reviewing and editing can minimize these concerns.

The most appropriate resume format is dependent on the individual and his or her experiences. If creating a resume for the first time, you may want to draft different formats to determine which works best for your history. Keep in mind that health educator job descriptions may not differ extensively and including achievements or other factors to identify uniqueness will help your resume transcend others. Similarly, health educator roles 
are skills based. As a new graduate, a listing of academic courses does not demonstrate application of the knowledge from that course. Indicating any practical application from coursework would be beneficial. In addition, highlighting any practical experiences such as an internship, volunteer experience, or student organization leadership that relates to health educator skills would provide the reviewer more information about your skills.

Likewise, any certifications or additional trainings should be included in the resume. If you are a CHES, that information should be highlighted in both the cover letter and resume. A review of job postings for academic positions between 1977 and 1992 showed an increased preference for CHES (Baker \& Cissell, 1994); this preferred qualification has most likely increased since then because of promotion of CHES by the National Commission for Health Education Credentialing (NCHEC) and other professional organizations such as American College Health Association and American Public Health Association (Coalition of National Health Education Organizations, 2001). However, in some settings, such as schools, the CHES may not be recognized and certifications through other organizations, such as the National Board of Professional Teaching Standards, may be more appropriate (Wooley, 2004). Emphasize certifications which are most related to the position, but all certifications related to health issues should be included (first aid, CPR, statecertified HIV counselor, etc).

Membership in national, regional and local professional organizations should also be listed. Unless there were additional responsibilities, such as being an officer or chairing a committee, listing the organization's name and dates of membership is sufficient.

Publications and presentations at conferences should also be included on the resume, even for those in non-academic settings. If the list is extensive, provide selected titles and indicate that the list is not exhaustive of all your work. Unless applying for an academic position, a summary of presentations and publications will be adequate.
Education experience, usually listed in reverse chronological order, must also be included, but the presentation will depend on the level of experience. New graduates may prefer to list this information immediately after the objective; whereas, seasoned professionals may include it at the end of the resume as their professional experience speaks more about their qualifications than their degrees.

Make sure that all information in the resume is an accurate statement of you, your skills and your experience. The resume is a selling tool, but you want to support all that you have listed and described. The field of health education is relatively small and any misrepresentation, intentional or not, could have long-term consequences.

\section{Writing \& Presentation Style}

While the cover letter is an opportunity to demonstrate your writing skills, the resume should consist of brief phrases that can be easily skimmed for highlights. Lengthy sentences or paragraphs make it difficult to recognize critical information. Most people only spend ten to 20 seconds reviewing a resume (Rockport Institute Ltd., 2003b), so you need to catch their attention and present the resume so that crucial elements are easily identified.

The use of power words to identify your accomplishments can help garner attention. These words and key phrases conjure positive images of action and responsibility. For example, "did a program for teens on tobacco" does not inspire a strong image; however, modifying the phrase to "facilitated a highly engaging tobacco program for high-risk youth” certainly presents a different image. Power words are often verbs; however, the rest of the phrase should be reviewed for possible improvement. Remember the resume is your tool to obtain an interview, so it is critical to present yourself strongly. Lists of power words (also called action words) can be found in many resume books or online (Rockport Institute Ltd., 2003a). In addition, use words appropriate for health education, specifically the area of this organization and position. Keeping up-to-date with journals, professional newsletters and 
listservs will help you identify key words and hot topics. Also, using the job description or other job postings may provide additional word and phrase options.

The overall appearance of the resume is also vital. A resume crammed with information, using a small font is difficult to read and will not likely be examined closely. The one-page rule for resumes is not as steadfast as in previous years. In general, one to two page resumes are acceptable, with two to three pages for those with substantial experience (Kennedy, 2003). Because the length limitation is more flexible, be sure to use white space to balance text. In addition, using bullets, dashes or indents can assist with organization and help the reader follow the information. Similarly, avoid using fancy fonts and colored paper that make content difficult to read.

Especially critical is proofreading the document. This step cannot be emphasized enough. Any typographical errors in grammar or spelling can be automatic disqualifications depending on the reader. Asking multiple colleagues and friends to review the cover letter and resume can reduce the risk of these errors. The more individuals who check the documents, the more likely something will be noticed and you will be able to change it before submitting it to a potential employer.

\section{Conclusion}

In order to create the best possible job application, the process is both time and energy consuming. One suggestion to minimize this effort is to constantly update your resume, even if you are not looking for a position. Keeping a current resume allows you the flexibility to make changes and toy with different formats, words and phrases without the time pressure of applying for an actual position. Many organizations require updated resumes with annual reviews and most of us, just print out last year's version. Take time this year to investigate ways to improve your resume and cover letter. Even if you never use the document, creating a cover letter and resume that highlights your skills and accomplishments can be rewarding in itself.

As with many projects, the time and effort involved in creating the best possible cover letter and resume is directly related to the end product. If you are serious about obtaining a new position, remember the cover letter and resume are the key mechanisms that potential employers will learn about you and your skills. Develop a job application packet that shows the world who you are and what you can do.

\section{References}

Baker, J. A., \& Cissell, W. B. (1994). Professionalism and academic job announcements in health education. Wellness Perspectives, 10(4), 40-48.

Coalition of National Health Education Organizations. (2001). The health education profession in the twenty-first century: Progress report 1995 - 2001. Retrieved February 15, 2005, from http://www.hsc.usf.edu/CFH/cnheo/downloads/21stCentury.pdf

Kennedy, J. L. (2003). Resumes for dummies (4th ed.). New York: Wiley Publishing, Inc.

Rockport Institute Ltd. (2003a). Add power to your resumer with powerwords. Retrieved February 28, 2005, from http://www.rockportinstitute.com/powerwords.html

Rockport Institute Ltd. (2003b). How to write a masterpiece of a resume; part 2 - How to knock the socks off a prospective employer. Retrieved February 8, 2005, from http://www.rockportinstitute.com/resume_02.html

The College of Wooster. (n.d.) Guide to writing a resume \& cover letter. Retrieved February 14, 2005, from http://www.wooster.edu/career/pdf/resumeandcoverletters2.pdf

Thomas, S. S. (1998). Employment in the non-profit sector: A primer for health educators. Eta Sigma Gamma: The Health Education Monograph Series, 16(1), 30-34.

Wooley, S. (2004, February 5). Re: Health Education Teachers - an outstanding opportunity. Message posted to Health Education Directory. Retrieved February 14, 2005, from http://www.kittle.siu.edu/hedir/listserv/2004/february/2\%2D05\%2D2004.htm 


\author{
Author Information \\ Sara B. Oswalt, MPH, PhD \\ University of Texas at San Antonio \\ Department of Health and Kinesiology \\ 6900 North Loop 1604 West \\ San Antonio, TX 78249 \\ Ph.: 210-458-6227 \\ Fax.: 210-458-5873 \\ E-Mail: sara.oswalt@utsa.edu
}

